\title{
The Application and Significance of Discourse Cohesion and Analysis in Practical Teaching of Foreign Language
}

\author{
Junxin Li \\ Department of Foreign Languages, Heze University, Heze 274000, Shandong, China
}

\begin{abstract}
The text cohesion and analysis have an important meaning in the practice of English teaching. It is the essential basis to get the clue of the text developing, deepen the understanding of the article, cultivating the students' ability of logical thinking, and do a good job at the important foundation to improve the quality of foreign language teaching. So, it's not an ignored strategy to master the theory of discourse cohesion and discourse analysis in the foreign language teaching, and the dependable guarantee to raise the quality of the foreign language teaching.
\end{abstract}

Index Terms-grammatical cohesion, lexical cohesion, discourse, reading teaching, writing teaching, translation teaching

\section{THE BASIC CONCEPT OF DisCOURSE COHESION AND ANALYSIS}

Cohesion is the basis of an article, is also the important form of showing the style and characteristics. It is linked to all kinds of term relationships, cohesion method generally includes two types, there are: grammatical and lexical cohesion.

Cohesion is a main steps of linking appropriate terms or grammar form into an article. In foreign language teaching, through the exploration and analysis of discourse, looking for the language in the form of cohesion and its regularity, to grasp the characteristics and the skills of the discourse cohesion, in order to let the learners to master the discourse context and ideological significance; familiar with the micro contact in discourse, improve the quality of foreign language teaching and learning efficiency and language application ability. Discourse is the language form of terms and sentences. It has the characteristics of the semantic coherence and tight logic. It shows the relative functions of the semantics, and makes the ingredients of discourse complement each other, constructing the unique aesthetic feeling of the language.

\section{A. Grammatical Cohesion}

The grammatical cohesion can be divided into four kinds of forms; (Hu Zhuanglin, P, 68-69) "such as reference, substitution, ellipsis, and connection. Care expresses the semantic relations with the grammar of pronouns speech etc," generally divided into personal care, instructive care and competitive care. Personal care is mainly used to reflect with possessive determiners and possessive pronouns. Instructions care is mainly composed of demonstrative pronoun and indicating adverbs, and comparative reference often reflect by the comparative of adjectives and adverbs. The textual features of reference across the sentence boundaries when it connects, allows author to use the short form of the demonstrative pronoun to express the objects with once mentioned or to be mentioned in the context of the content, so that the discourse can be concise and compact. Alternative means with indefinite pronouns, such as; "do", "one", "so" etc, to take place ingredients occurring in the context. The index relationship between substitution and replacement parts can make the sentences in discourse closely linked together. Omitted is a special form of alternative, alternative terms have been omitted. Effect of substitution and omission is to highlight the main meaning, to avoid duplication, maintain close relationship between contexts. Connection is a means which using connection components reflect what logical relationship between different components in discourse. The connection components often use the transitional words with some logical relationship such as: time, cause and condition etc. Connecting words like road signs, will guide readers to understand the semantic relationships between sentences, and grasp the connotation of the discourse.

\section{B. Lexical Cohesion}

Lexical cohesion appearing in discourse just means some semantic relationship between partial terms, including reproducibility and co-occurrence. Reproducibility relationship of the vocabulary refer to a particular word in the original word, synonyms, approximate meaning word, antonyms, hyponyms, general word and other forms reappear in the discourse. Sentences in a discourse link each other by this relationship of reproducibility. Co-occurrence relationship of words means the tendency of the common occurrence, such as: "University", and "professor", "Spring" and "Flowers", and so on. Lexical cohesion is the most prominent, the most important form of convergence. They are the major means of creating discourse forms, about account for more than half of quantity of discourse cohesion. 
Accurate understanding of the discourse is also inseparable from the analysis of the discourse. Discourse analysis should pay attention to two aspects: First, the structure of the text, the second is function of discourse components. The discourse is the language unit which structured with sentences, structure is very complex; discourse is a semantic unit, its relationship with sentences is relationship of embodiment, which means the structure of the sentences come to embody the semantic content. In the teaching practice of translation, if we want to form a discourse that fit the need in the target language society, it should choose the language resources from top to bottom and with a goal, in order to rewrite the whole article. The description must focus on the semantic convergence between the structure and characteristics of sentences and the different components.

We know that any language has its own voice system, syntax system and semantic system. English is a form of "shape-legitimate" language of Indo-European, commonly used in various forms of conjunctions, phrases, clauses and subordinate clause, focusing on the dominant convergence and sentence form, focus on structural integrity. But Chinese is "legal meaning" language, rarely use or never use the means of "shape-formed", always use the hidden meaning to link with others. Especially pay attention to the orders of logic affair, the functions and its meaning. It should say that English stresses on well-formed, and Chinese stresses on the form of meaning formed, we must pay more attention to this feature when we carry on the discourse analysis.

From the form of cohesion, "shape legitimate" of English link up the sentences with conjunctions by syntactically. The "legal meaning" of Chinese relies on the sense of convergence, but not necessarily dependent on the conjunctions. So, conjunctions used in English have a very high frequency.

The grammatical cohesion way in English is phenotype, and Chinese is stealth. Chinese expression flexible, many logical relations rely on meaning rather than grammar. The omitted sentence is just to convey its ideas, without thinking of grammar or the logical means, the omitted words were used less than English. Chinese always use the repetition of original words to realize the textual cohesion.

\section{The Discourse COHESION AND ANALysis In TEACHING OF READING}

In the traditional English teaching of reading, teachers have more emphasis on vocabulary, sentence structure, grammar, and pay less than the analysis of discourse structure and coherence, lead to students to master the only fragmented knowledge, it's difficult to grasp the article content and structure as a whole. Discourse theory suggests that cohesion is the clue of discourse coherence, through analyzing the text, we can make students to accurately grasp the main thinking and structure of the article. Statement smoothly between smoothly lexical chain between sentences and words is advantageous to complete paragraph meaning, also be the assurances that students can understand the article well.

Discourse is a component of sentence connections, it is not a simple arrangement of works, but in a certain language style to each part of the article using the skill originally combined into a whole. Mode plays an important role in the discourse construction, and discourse analysis can solve many problems that they are not solve with grammar. Reading, therefore, should not only pay attention to the words, phrases, sentences, grammar structure, also from discourse level, giving a macro analysis of the content of the article structure, cultivate students' logical thinking, improve their comprehensive reading ability.

Reading teaching only analyze the words and sentences is not only obtain a good result, only seize the discourse form and content, combining with environment of language usage to understand and grasp the discourse can seize the essence. Clearly explained in the form of the language knowledge, to analyze use of cohesive devices, to create discourse coherence method, combined with the learned articles related to the cultural and social knowledge, analysis, together with the students in the process of cognitive inference, induction, summarizes the knowledge of the discourse and training the students' ability of thinking and language use. Teachers should earnestly practice more, provide students with more opportunities to explore and exchange knowledge. Usually the process for imparting new knowledge is: to inspire interest in ways to import new lesson, speculate clue of the story development, let the students read the text quickly, and understand the content and structure of the article, find out the related problems classify each section of the story and summarizes the central idea, give the overall understanding exchange activities such as organization and retelling, discussion, to deepen understanding of the article do the training of language skills, discuss the cohesive devices and writing skill of articles, style and rhetoric, to understand passages with valuation. This is good for student's ability to obtain overall information, improve their subjective initiative and the ability of independent thinking, and the communicative ability with foreign language.

In teaching of discourse analysis, "teachers should fully mobilize students' learning enthusiasm and subjective initiative, treating the language analysis as the key step in the training students' language ability to grasp."(Jia Lijuan, P, 54) It can inspire students' thinking, but also can help students analyze cohesion and coherence between sentences, understanding communication function of discourse. In text reading, students should analyze, reasoning, and summarize the process of writing the text actively, focusing on the learning content. In this way, it can help to improve the students' ability and communication skills. Teachers ought to explain the important vocabulary and grammatical structure in learning, and try to create good communication atmosphere for students, exercise their ability of language application.

Reading teaching in classroom should stimulate students' interest and the language training should pay attention to the language content and development of communication skills. In reading teaching, considering the actual situation of 
language knowledge level, the knowledge of language, we must give detailed explaining, especially to the obstacles of understanding the grammar and sentence structure. As far as possible use the learned language to skim through knowledge and capture the important language clues in discourse teaching, have an overall impression to the article, segment the article into some sections and find the central idea and important information. We should be good at understanding the intention of the article, carries on deep analysis to each other, so that the students based on the content, speak out their own opinions, and evaluative understanding.

English articles have all kinds of different styles and characteristics: generally speaking, the narrative is to pay more attention to the experience of the event. Description lays particular stress on description of emotion. Its language is vivid, and exposition especially notices the organized, more objective and concise language style, thesis lays stress on logic rationality in the development of things.

"The meaning of the statement understanding depends on discrimination of word and rhetorical devices."(Zeng Qingyong, P, 23-24) The emergence of new words will reduce the speed of reading, hinder the understanding of sentence meaning and discourse. Teachers can guide students by means of word formation or use context to a ascertain meaning, inspire the student from the part of speech, the rhetorical features, comparison of statements, restatement, the word with the relationship of context, to differentiate and analyze words according to the author's style and aims of selecting words. In the use of rhetorical figures is also a difficulty. The rhetorical use of English and Chinese have some similarities and intersection, but sometimes, English and Chinese speakers have completely different cultural schemata, lexical connotation and denotation is a big difference. In rhetoric teaching, the teacher not only requires students to identify and right understanding of the rhetoric meaning, still should guide students to compare Chinese and Western cultural schemata, to improve the sensitivity of cross-cultural communication. At the same time, we should help students analyze the rhetoric of the ideographic function, the connection function and aesthetic function in the discourse.

Discourse analysis in reading teaching emphasizes the discourse, as a whole, content, significance, complied with people's cognitive process of thinking, to improve students' reading comprehension ability and cultivate students' ability in all aspects of language use. It narrowed the dominant position of the teacher in the classroom, the whole teaching process centered on student activities. Practice has proved, through discourse analysis teaching, teachers can train the student quickly and accurately grasp the basic content of discourse and the central idea, find out the author's thinking, to understand how the author is expressed the ideological content through the language structure. Discourse analysis teaching plays a very important role to improve students' reading ability, cultivate the students' analysis, induction, integration and inference ability and the ability of preliminary language communication, it is the benefit on helping students to develop good reading methods, to improve their reading comprehension level, improve the quality of reading teaching.

\section{The Discourse COHESION AND ANALYSis IN Writing TEACHING}

In the current writing teaching of foreign language, we noticed that the students' grammar in writing is basically correct, sentences are also reasonable and smooth, and the structure of article is disorder, poor works, lack of logic with the statement and paragraphs, the lack of discourse coherence. In writing teaching, therefore, do not think highly of the learning and use of discourse cohesion theory, and improve students' ability in discourse construction, it is difficult to train out a high level of writing skills.

To do this, first we should realize the importance of textual study, and teach theory of the discourse cohesion systematically, and applied to the student the teaching of writing. Make students attach importance to the overall structure of the article and the analysis of the ideological content, making clear to the clue of text development, realize the entire train of thought, comprehend the specific role of cohesion in discourse construction. Also, enlarge students' vocabulary, guide students to use a variety of terms, such as: the original words, synonyms and approximate meaning words, hyponyms and summary words etc, all kinds of ways in lexical cohesion to express, to improve language dull phenomenon. Teachers should systematically, in a planned way to do more training of writing coherence knowledge. Just as; design filling blanks, make sentences, interference into paper and other special exercises, imitation of creative writing, such as rewriting, repeat, copy writing, paying special attention to composition of overall planning and the use of cohesive devices.

It is the basic skills of foreign language learning in English writing, attaches great importance to the discourse of knowledge learning, enable students to master the article of the entire building and writing skills.

Students' errors of discourse also include the lack of discourse knowledge, the influence of traditional grammar teaching mode and the lack of various factors, such as the side effects of their mother tongue. Students don't have knowledge of discourse, don't know how to construct the discourse; Superficial understanding for word cohesion and composition mode, misuse and abuse related vocabulary; Coupled with the interference of mother tongue, and could not clearly differentiate the use of punctuation in English and Chinese, result in the sentence structure is chaos. The traditional foreign language teaching too much emphasis on accurate grammar, ignoring the students' creative ability and logic of discourse coherence, the composition expression became a vocabulary list and packing. Therefore, in teaching of English writing, we should pay special attention to: (1) the differences of two cultures, we ought to strengthen the cultural comparison education, avoid the misunderstanding of awareness brings the error use of culture. 
English culture is expressed directly and Chinese culture has many hidden song. (2) Arouse the students' creative consciousness, cultivate the continuity thinking of foreign language application. (3) Cultivate macro overall consciousness in discourse, avoid writing indiscriminately according grammar. (4) Reform the standard of evaluation, strengthen the ability of discourse coherence. "In the writing teaching should fully understand the theory of discourse coherence and cohesion means, in-depth study of the teaching methods, to explore the students' awareness of discourse and the expression and rules of discourse errors, find out the countermeasures to solve the problem." (He Xuegui, P,56)

Any type of discourse have specific stylistic features, stylistic analysis focus on the language description of text, analyzing the appropriateness of the selected language ingredient as well as the effect of specific problems, so as to achieve the aim of appreciation on text. Stylistic analysis carred on is in pronunciation, writing, vocabulary, grammar, discourse structure, and other aspects. The focus is the language characteristics of analysis of stylistic significance and aesthetic value, from the perspective of the typical features of language, explore the author's pragmatic intention and pragmatic effect, in order to achieve the purpose of appreciation and follow, improve their writing level. So, analyze the appropriateness of language elements selected by author and its specific stylistic rhetoric effect is also an important part of the writing teaching.

In the teaching of English writing, teachers also need to use discourse analysis method, from the perspective of textual macro, pays attention to the subject of essay writing, paragraph structure, sentence relations and the writing features, let the student maximum master the links and skills in writing. Writing teaching is a process of continuously explore. We must constantly explore the teaching ideas, new methods, cultivate students' logical thinking, independent thinking and innovation ability. Practice has proved that the guidance of discourse analysis in writing teaching, can strengthen the students' interest in learning, improve their writing skills and application ability. Greatly promote the students' learning and mastery of the language and culture.

\section{COHESION AND ANALYSiS In TRANSLATION TEACHING}

In translation teaching practice, the traditional habit in sentences is as basic unit, we find out the fusion point through the real meaning of terms and expressions of two kinds of culture, corresponding to the translation of sentence by sentence, word for word. Researching of discourse theory can help us out of error place from the discourse context and words of translation. Familiar with cohesion theory, translation teaching can more accurately convey the original meaning. In translation teaching, teachers should consider the following factors:

(1). Selecting material of translation should pay attention to discourse as the unit, before translation you should read the whole text, and then use the cohesion theory to analyze, form the overall consciousness of discourse, and grasp the original essence, guarantee the translation faithful to the original one. (2). First, choose the model translation to analyze, comparing the difference between the cohesive ties in both Chinese and English, make out the measures to deal with the differences. Such as: English pronouns is in textual cohesion are often in the former, the parts that stood for is at the last. And the Chinese is just the opposite. The orders must adjust when we translate them. Mastered the comparison and analysis may improve the translation skills. (3). You should pay attention to the core words of cohesion when reading the original article, if some words are difficult to understand, you can be through the analysis of repetition of words and co-occurrence relations to master its precise meaning. In translation, English and Chinese different patterns of lexical cohesion ought to do appropriate conversion, reappearance original text rhetoric effect as far as possible, to keep language style of the original.

When reading the original and the translation, you should analyze the discourse. After analysis of the results, we can compare them, lay a foundation for the accurate translation. Therefore, translation should have the consciousness of discourse. The discourse awareness can make us pay attention to the integrity and indivisibility of the chapter in translation research and practice, achieve the function of discourse communication and communicative intention, governance the observation and study of discourse level. "Translation despite the style difference, but the translation by understanding and convey associated composition is out of question." (Yu Gaofeng, P, 41-42) In the process of translation, and understanding, grasping of discourse cohesion to the translation of the original has a vital role.

On the original analysis, we should delve into the syntax and discourse structure. Analysis of the discourse structure is an important part of the translation, and in the link mode is also easy to make mistakes, translator can complete understanding of the original exactly, if you want to avoid the structure in vague or logical confusion when translation, must hold the discourse structure of the article well both in English and Chinese.

Transmission is the center of the translation purpose. In understanding, concern the core is the author, in the process of conveying, attention of the object is the reader, you should always think of using acceptable language which the readers can admit to express the author's intention and the original content. Therefore, the translator should consider of cohesive devices in both Chinese and English languages, pay attention to the differences between the two kinds of cohesion, ensure of the translation conforms to the habits of target language.

Discourse translation is the current trend on translation study, in discourse translating, cohesion is the important features and forming conditions. In Chinese-English translation, the cohesion exchange making semantic coherence is the key step in the translation. Cohesive devices between English and Chinese has a big difference, therefore, we need to in-depth study of cohesive devices and means, grasp its regularity, for translation accurately convey the original ideas. Therefore, cohesion and analysis are very important in understanding the source and formation. 


\section{The Significance of Discourse Analysis in Teaching Practice}

In traditional foreign language teaching in the past, we only attach importance to language forms, rarely stand in the Angle of discourse or context of article to make a detailed analysis, the students don't grasp the thread of the article and implied meaning. They only read and understand the article word by word, the comprehensive ability of students' foreign language learning cannot be formed. If they have learned the cohesion theory, they can make full use of the skills on the learning of discourse. Thus, their basic language knowledge and the abilities of comprehensive application can be improved significantly.

M. Halliday (P, 99), British linguist points out that discourse analysis is the interpretation of the discourse, to the discourse genre, central idea and structure do first assumption in advance, give an analysis to the discourse style and structure; Compare our prediction with the author's entire agreement, tell their different advantages and disadvantages. Pay attention to the discourse of cohesion, before and after the echo, coherently of the logical relationship, combined with the cultural background, a comprehensive grasp the profound meaning of the text, so as to make comprehensive evaluation to discourse.

When we learn the genre analysis that we should let students understand the knowledge of article genre, such as: classification, characteristics and components, then to forecast the discourse genre, speculation of overall planning to reveal the central idea, again carries on the contrast and analysis, find out the argument.

Theme is the enrichment and generalization of full text content, expresses the author's writing intention, especially in the topic sentence of a paragraph. You should think to find it out when reading, in order to get to know the theme idea of the discourse exactly.

Structural analysis is influenced by discourse genre, theme; the structure is not the same. British linguist Michael Hoey divided the discourse into many varieties: include general type - points above, (after summary set example; detail explanation after whole); Contrast - match type, (in contrast similarities and differences to highlight the central issue.) ; Problem - solving. (put forward the existing problems first, and then choose solution). In practical teaching, referring to the mode of discourse, inductive each paragraph and hierarchical to the title, understand the overall structure of the article by different administrative levels, comparing with contrast methods, pave for the theme to highlight the structure of the discourse.

Halliday and Hasan believed that cohesion is the language resources in each of language, and it can associate with this or that portion of the discourse. At the same time, they divided the cohesion into such grammatical tapes: the reference, ellipsis, substitution, conjunction etc, and lexical cohesion can be divided into the methods: recreate and collocation.

When we read and analyze the relationship of sentence, we should pay an attention to the use of conjunctions. Conjunction can reflect all kinds of the logic relationship in discourse, the logical relationship is a link to form the discourse network. We usually have four patterns: adding, turning, causal and time.

Liu Chensheng $(\mathrm{P}, 199)$ pointed out that language is the carrier of culture, it is not independent existence. Therefore, if you want to master a foreign language, you must consciously understand and accept the rich culture of a language. Discourse is the product of social and cultural context, it reflects a certain social intention and cultural characteristics. Therefore, discourse is associated with a particular cultural significance. In the discourse analysis, combining with the cultural background of the article, is more advantageous to the students for understanding the theme of the thought and moral that reveals in the article.

In a word, to cultivate students' ability in discourse analysis, in class, we should train students' abilities of summarizing the ideas of article, retelling, paraphrased, rewrite the plots of text and the expression ability of content; Outside the classroom, students are encouraged to read all kinds of genre of materials; meanwhile, you should pay attention to the forecast and analysis of the cohesion relationship, thematic structure and cohesion genre, consciously train the ability of discourse analysis. Strengthen students' understanding of the social culture, economic development and historical origin in the target language, and expanded the aspect of knowledge; improve the discourse analysis and language application ability.

\section{CONCLUDING REMARKS}

To sum up, the theory of discourse cohesion and analysis is an important means of textual research. In the teaching, rationally use the theory of discourse cohesion and analysis, you can better help students grasp the chapter structure of the article in general, improve the level of the students' reading, writing and translation. This way of teaching for teachers also put forward the corresponding requirements. Teachers must have a comprehensive and thorough understanding of this theory and consciousness of discourse analysis. And we should be good at exploring the teaching method in teaching and applied to the practice. Continuously explore and improve them, then you can comprehensively improve the student's application ability of foreign language in the teaching.

\section{REFERENCES}

[1] He Xuegui. (2009). Discourse Coherent Cohesion Theory and English Professional Writing Teaching. Journal of Huanggang Normal University 1, 56 
[2] Zeng Qingyong, Wang Fei. (2008). Lexical Cohesion of College English Reading Teaching Enlightenment. Journal of Qiqihar Medical College 8. 23-24

[3] Halliday. M. A.K. (1985). An Introduction to Functional Grammar, London: Edward Amold.

[4] Hu Zhuanglin. (1994). The Cohesion and Coherence of Discourse. Shanghai, Shanghai Foreign Language Education Press.

[5] Jia Lijuan, Duan Xiaoli. (2006). System Function syntax Discourse Analysis in the Application of English Extensive Reading Teaching. Journal of Beijing City University 4, 54.

[6] Yu Gaofeng. (2009). Discourse Cohesion Coheren and translation. Language and Translation Journal 4, 41-42.

[7] Liu Chensheng. (1999). Teaching Discourse Linguistics. Shanghai, Shanghai Foreign Language Education Press.

Junxin Li was born in Heze, Shandong, China in 1955. He received his bachelor degree from Tonghua Normal University in 1982.

He is currently an associate professor in the department of foreign languages, Heze University, Heze, China. His research interests include English applied linguistics and sociology. He has published many research papers and some works. 